\title{
KNTC1 is differentially expressed in lymph node metastasis in human breast cancer.
}

Shahan Mamoor, $\mathrm{MS}^{1}$

${ }^{1}$ shahanmamoor@gmail.com

East Islip, NY USA

Metastasis to the brain is a clinical problem in patients with breast cancer ${ }^{1-3}$. Between the breast and the brain reside the secondary lymphoid organ, the lymph nodes. We mined published microarray data ${ }^{4,5}$ to compare primary and metastatic tumor transcriptomes for the discovery of genes associated with metastasis to the lymph nodes in humans with metastatic breast cancer. We found that kinetochore associated 1, KNTC1, was among the genes whose expression was most different in the lymph node metastases of patients with metastatic breast cancer as compared to primary tumors of the breast. KNTC1 mRNA was present at increased quantities in lymph node metastases as compared to primary tumors of the breast. Importantly, expression of KNTC1 in primary tumors of the breast was correlated with patient overall survival, in lymph node negative patients but not in lymph node positive patients. Modulation of KNTC1 expression may be relevant to the biology by which tumor cells metastasize from the breast to the lymph nodes and the brain in humans with metastatic breast cancer.

Keywords: breast cancer, metastasis, brain metastasis, central nervous system metastasis, lymph node metastasis, kinetochore associated 1, KNTC1, systems biology of breast cancer, targeted therapeutics in breast cancer. 
One report described a 34\% incidence of central nervous system metastases in patients treated with trastuzumab for breast cancer ${ }^{2}$. More recently, the NEfERT-T clinical trial ${ }^{6}$ which compared administration of either neratinib or trastuzumab in conjunction with paclitaxel demonstrated that in a randomized, controlled setting, in breast cancer patients treated with neratinib, not only was the incidence of central nervous system recurrence significantly lower, the time to central nervous system metastasis was significantly delayed as compared to patients administered trastuzumab ${ }^{6}$. The alarmingly high rate of central nervous system metastasis described, as well as data, both anecdotal ${ }^{2}$ and from a randomized, controlled setting ${ }^{6}$ illustrating that treatment with trastuzumab may be associated with these events demands an enhanced understanding of the transcriptional makeup of brain and lymph node metastatic tissues to support identification of therapeutic targets, whether they are treatment related or not. The lymph nodes reside between the breast and the brain. We performed a global comparative analysis of lymph node metastatic tumor tissues in patients with metastatic breast cancer, compared to primary tumors and normal breast tissues ${ }^{4,5}$. We discovered transcriptome-wide differential expression of the gene encoding kinetochore associated 1, KNTC1, in lymph node metastatic tissues of patients with metastatic breast cancer.

\section{Methods}

We used datasets GSE10893 ${ }^{4}$ and GSE124648 ${ }^{5}$ for this global differential gene expression analysis of brain metastatic breast cancer in conjunction with GEO2R. GSE10893 was generated using Agilent-011521 Human 1A Microarray G4110A technology with $n=71$ primary breast tumors and $n=7$ lymph node metastases from patients with breast cancer; analysis was performed using platform GPL887. GSE124648 was generated using Affymetrix Human Genome U133A array technology with $n=10$ normal breast tissues and $n=44$ lymph node metastases from patients with breast cancer; analysis was performed using platform GPL96. The Benjamini and Hochberg method of $p$-value adjustment was used for ranking of differential expression but raw $p$-values were used to assess statistical significance of global differential expression. Log-transformation of data was auto-detected, and the NCBI generated category of platform annotation was used. A statistical test was performed to evaluate whether KNTC1 gene expression was significantly different between primary tumors of the breast and lymph node metastases in humans with breast cancer using a two-tailed t-test. For Kaplan-Meier survival analysis, we used the Kaplan-Meier plotter online tool ${ }^{7}$ for correlation of KNTC1 mRNA expression levels with overall survival in $n=726$ lymph node negative breast cancer patients and $n=452$ lymph node positive patients.

\section{$\underline{\text { Results }}$}

We performed global comparative transcriptome analysis of metastatic tumor tissues of patients with metastatic breast cancer using published microarray data ${ }^{4,5}$ to describe the transcriptional landscape of metastasis in human breast cancer in an unbiased fashion and for the discovery of novel therapeutic targets.

\section{KNTC1 is differentially expressed in the lymph node metastases of patients with metastatic breast cancer.}

Through blind, systems-level analysis of published microarray data ${ }^{4}$, we identified kinetochore associated 1, encoded by KNTC1, as a differentially expressed gene in the lymph node metastatic tissues of humans with breast cancer (Table 1). When sorting each of the genes expressed in lymph node metastases based on significance of difference as compared to primary tumors of the breast in patients with breast cancer, KNTC1 ranked 73 out of 18034 total transcripts (Chart 1), equating to 99.6\% differential expression. Differential expression of KNTC1 in the lymph node metastases of patients with metastatic breast cancer was statistically significant (Chart $1 ; p=4.76 \mathrm{e}-04$ ). 
We queried a second microarray dataset ${ }^{5}$ to attempt to validate transcriptome-wide differential expression of KNTC1 in the lymph node metastases of patients with metastatic breast cancer. We again observed KNTC1 to be among the genes expressed most quantitatively differently in the lymph node metastases of patients with metastatic breast cancer as compared to primary tumors of the breast. When sorting each of the genes expressed in lymph node metastases based on significance of difference as compared to normal breast tissues, KNTC1 ranked 1924 out of 22283 total transcripts (Chart 2), equating to $91.4 \%$ differential expression. Differential expression of KNTC1 in the lymph node metastases of patients with metastatic breast cancer approached the level of statistical significance (Chart 2; $p=5.25 \mathrm{e}-05)$.

KNTC1 is expressed at higher levels in the lymph node metastases of patients with metastatic breast cancer.

We obtained exact mRNA expression levels for KNTC1, in primary tumors of the breast and in lymph node metastases of patients with metastatic breast cancer to determine direction and statistical significance of change in KNTC1 expression in lymph node metastatic tissues. We observed significantly increased expression of KNTC1 in the lymph node metastases of patients with breast cancer as compared to primary tumors of the breast: KNTC1 was expressed at $-1.31 \pm 0.56$ arbitrary units (AU) in primary tumors of the breast, while it was expressed at $0.62 \pm 0.44 \mathrm{AU}$ in lymph node metastatic tissues (Figure 1). The difference in KNTC1 mRNA levels between primary tumors of the breast and lymph node metastases was statistically significant (Figure $1 ; p=0.00054$ ).

\section{KNTC1 expression is significantly correlated with survival outcomes in human breast cancer.}

We performed Kaplan-Meier survival analysis ${ }^{7}$ in $n=452$ lymph node positive breast cancer patients and $n=726$ lymph node negative breast cancer patients, to evaluate whether KNTC1 tumor expression was correlated with survival outcomes in breast cancer and to evaluate whether lymph node status influenced correlation of KNTC1 tumor expression with survival outcomes in human breast cancer. We observed a correlation between primary tumor expression of KNTC1 and overall survival (OS) in lymph node negative breast cancer (Figure 2). Lymph node negative patients whose primary tumors expressed low levels of KNTC1 possessed median OS of 35.27 months, while lymph node negative patients whose tumors expressed high levels of KNTC1 possessed median OS of 59.88 months. This difference in OS based on KNTC1 tumor expression in lymph node negative patients with breast cancer was statistically significant (Figure 2, Chart 3; logrank $p$-value: 0.0012 (hazard ratio: 0.57 (0.41-0.81))). Thus, overall survival based on KNTC1 primary tumor expression was markedly longer in lymph node negative patients than in the general breast cancer patient population. KNTC1 primary tumor expression was not significantly correlated with overall survival outcomes in lymph node positive patients (Figure 2, Chart 3; logrank $p$-value: 0.15 ; hazard ratio: $1.29(0.91-1.81)$ ).

Thus, by mining published microarray data ${ }^{4,5}$ in an unbiased and systematic fashion, we identified KNTC1 as among the genes whose expression was most different, transcriptome-wide, in the lymph node metastases of patients with breast cancer, when compared to primary tumors; we observed significantly increased expression of KNTC1 in lymph node metastases as compared to primary tumors of the breast. Further, we found a significant correlation between KNTC1 expression and patient survival outcomes, as overall survival was significantly greater in patients whose primary tumors expressed low levels of KNTC1 as compared to patients whose primary tumors expressed high levels of KNTC1, and this correlation was was significant in lymph node negative patients but not in lymph node positive patients. 


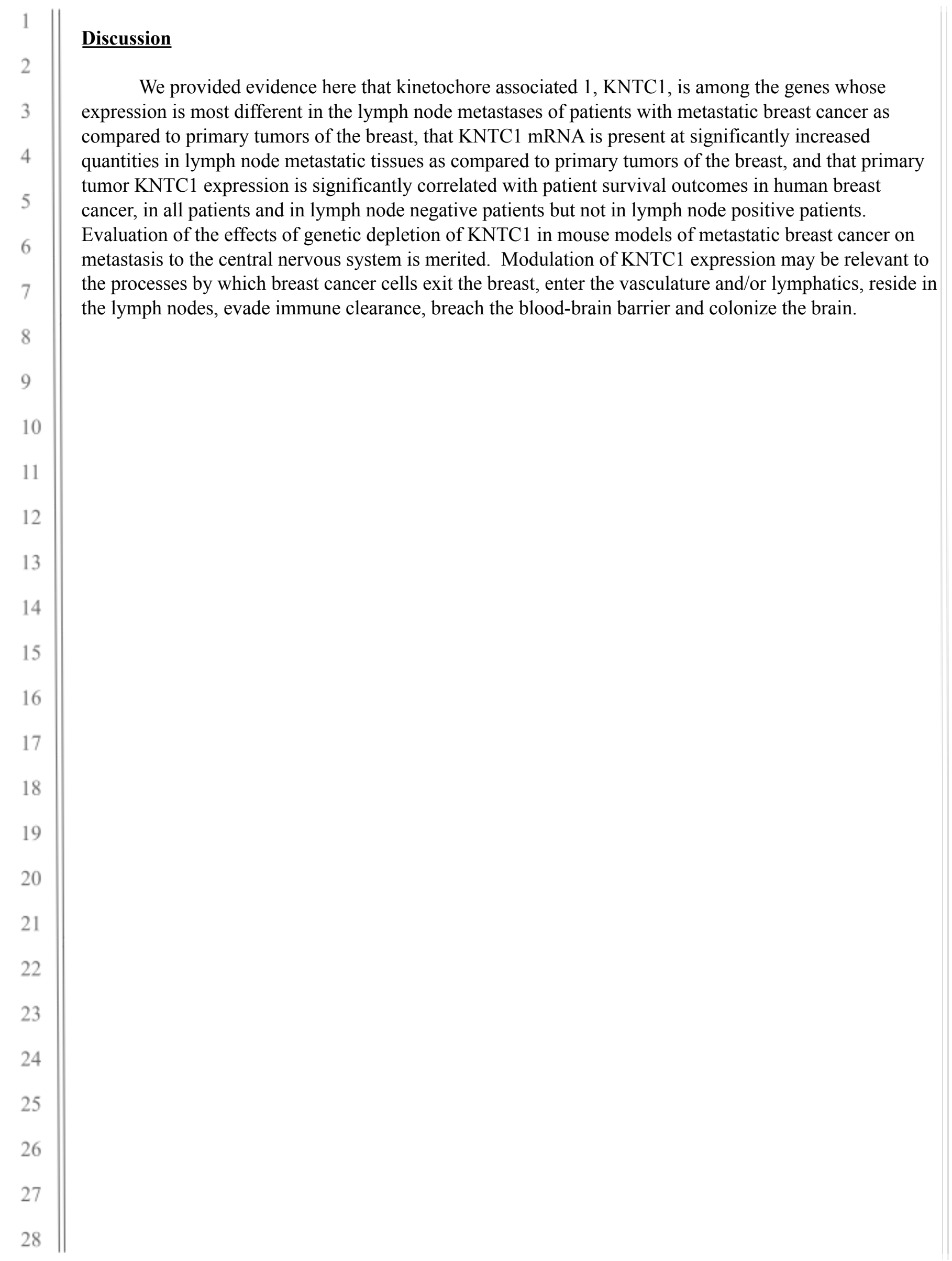




\section{$\underline{\text { References }}$}

1. Lin, N.U., Amiri-Kordestani, L., Palmieri, D., Liewehr, D.J. and Steeg, P.S., 2013. CNS metastases in breast cancer: old challenge, new frontiers.

2. Bendell, J.C., Domchek, S.M., Burstein, H.J., Harris, L., Younger, J., Kuter, I., Bunnell, C., Rue, M., Gelman, R. and Winer, E., 2003. Central nervous system metastases in women who receive trastuzumab-based therapy for metastatic breast carcinoma. Cancer, 97(12), pp.2972-2977.

3. Tsukada, Y., Fouad, A., Pickren, J.W. and Lane, W.W., 1983. Central nervous system metastasis from breast carcinoma autopsy study. Cancer, 52(12), pp.2349-2354.

4. Weigman, V.J., Chao, H.H., Shabalin, A.A., He, X., Parker, J.S., Nordgard, S.H., Grushko, T., Huo, D., Nwachukwu, C., Nobel, A. and Kristensen, V.N., 2012. Basal-like Breast cancer DNA copy number losses identify genes involved in genomic instability, response to therapy, and patient survival. Breast cancer research and treatment, 133(3), pp.865-880.

5. Sinn, B.V., Fu, C., Lau, R., Litton, J., Tsai, T.H., Murthy, R., Tam, A., Andreopoulou, E., Gong, Y., Murthy, R. and Gould, R., 2019. SET ER/PR: a robust 18-gene predictor for sensitivity to endocrine therapy for metastatic breast cancer. NPJ breast cancer, 5(1), pp.1-8.

6. Awada, A., Colomer, R., Inoue, K., Bondarenko, I., Badwe, R.A., Demetriou, G., Lee, S.C., Mehta, A.O., Kim, S.B., Bachelot, T. and Goswami, C., 2016. Neratinib plus paclitaxel vs trastuzumab plus paclitaxel in previously untreated metastatic ERBB2-positive breast cancer: the NEfERT-T randomized clinical trial. JAMA oncology, 2(12), pp.1557-1564.

7. Györffy, B., Lanczky, A., Eklund, A.C., Denkert, C., Budczies, J., Li, Q. and Szallasi, Z., 2010. An online survival analysis tool to rapidly assess the effect of 22,277 genes on breast cancer prognosis using microarray data of 1,809 patients. Breast cancer research and treatment, 123(3), pp.725-731. 


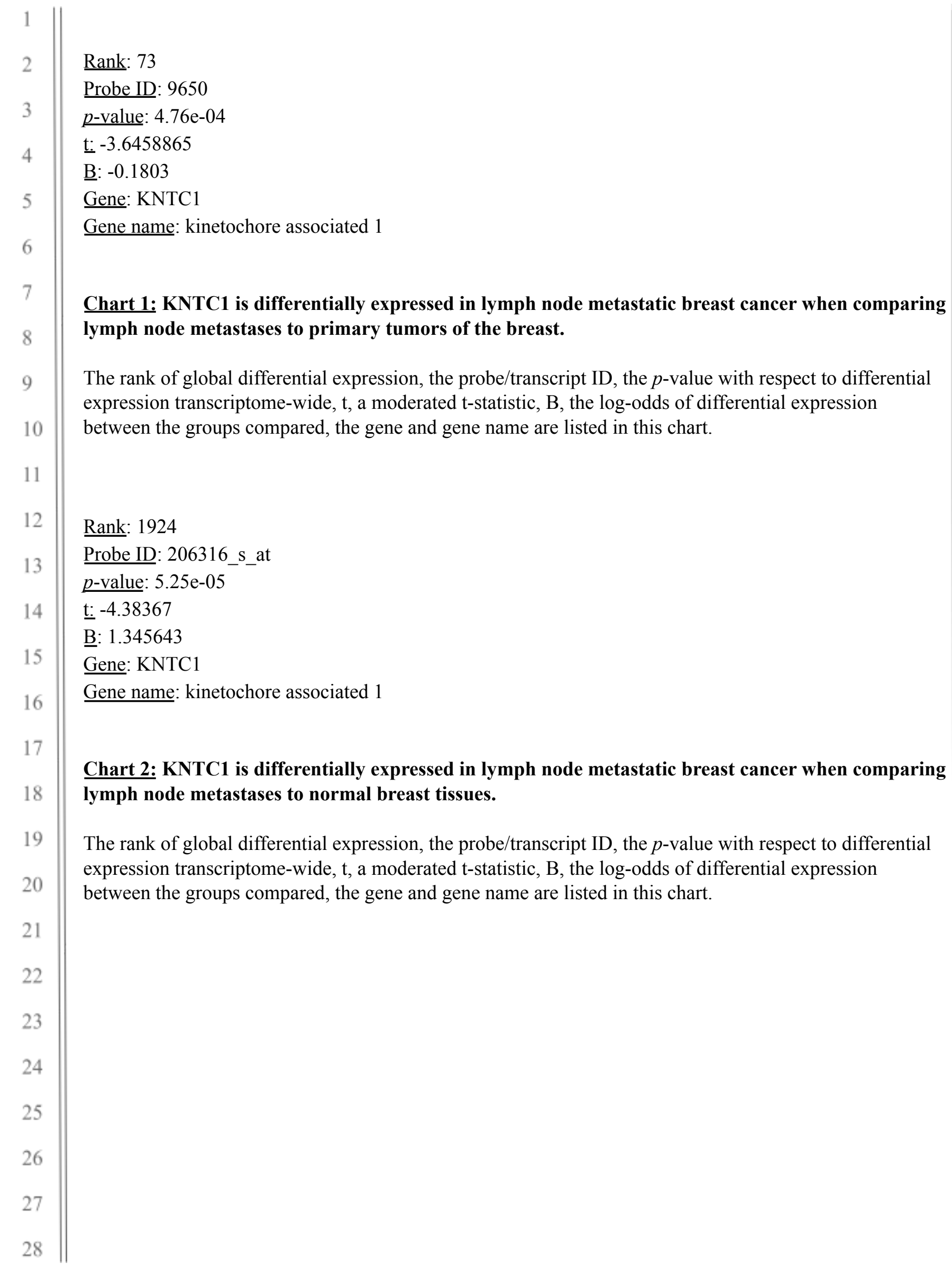




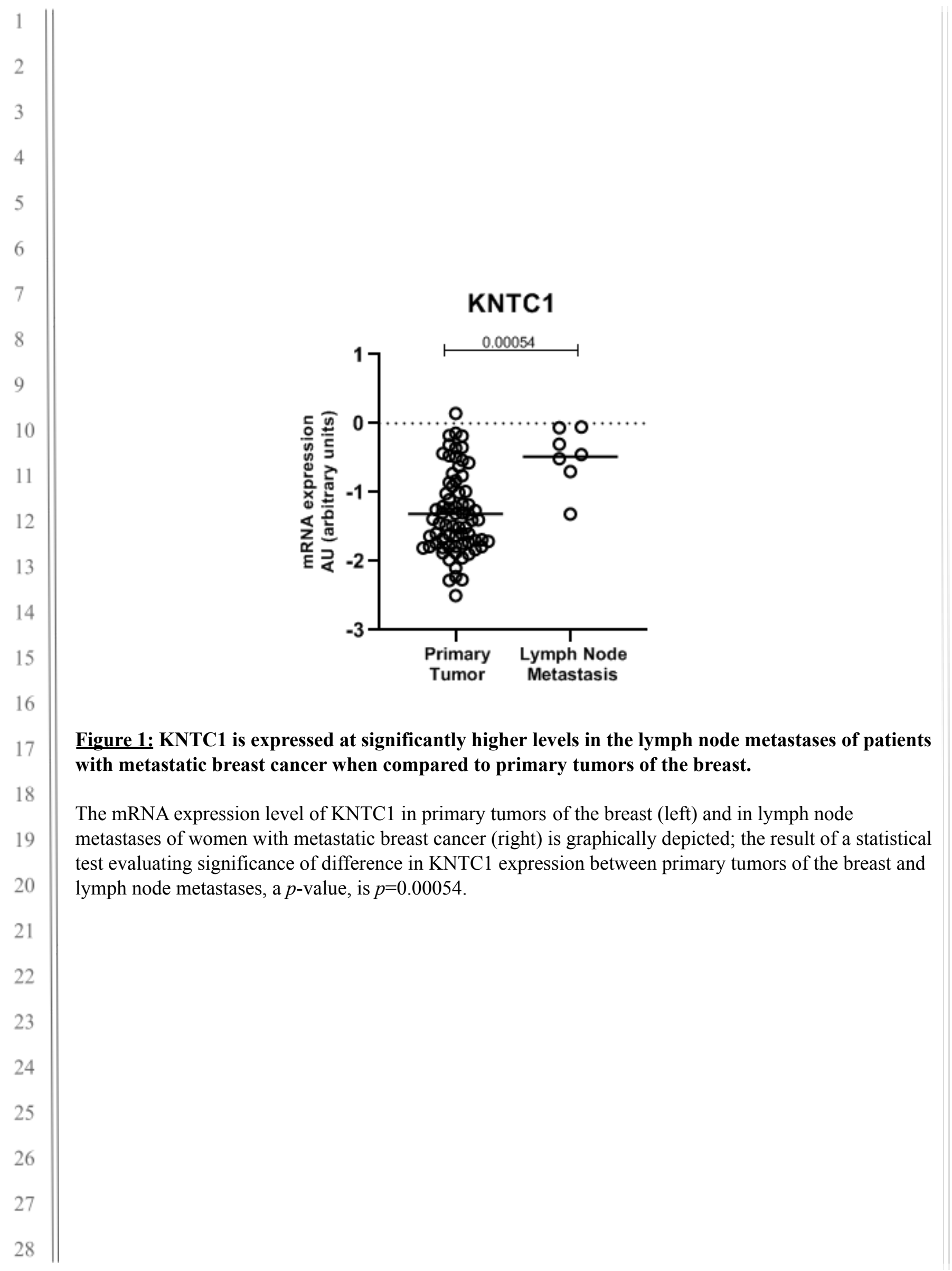




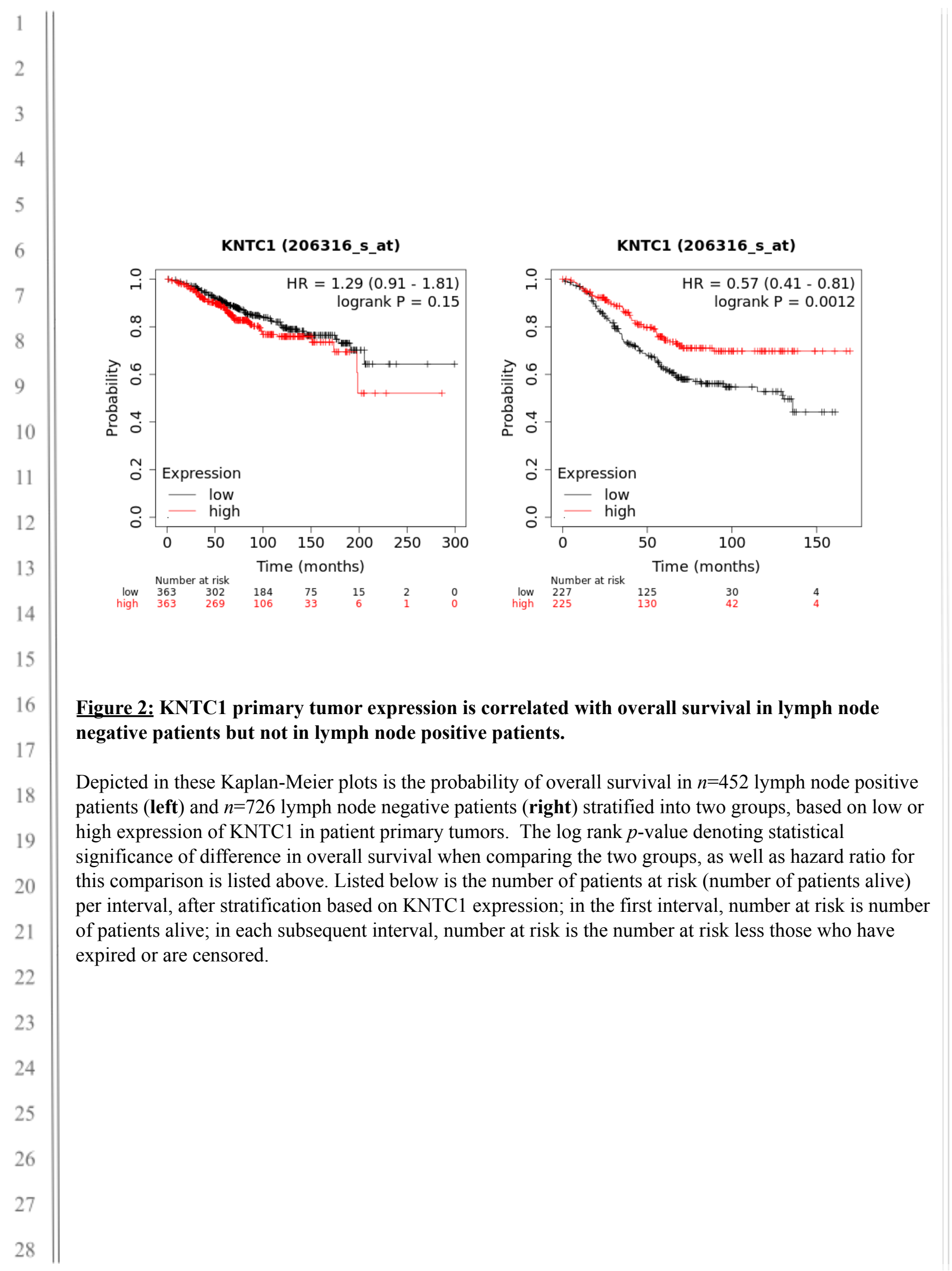




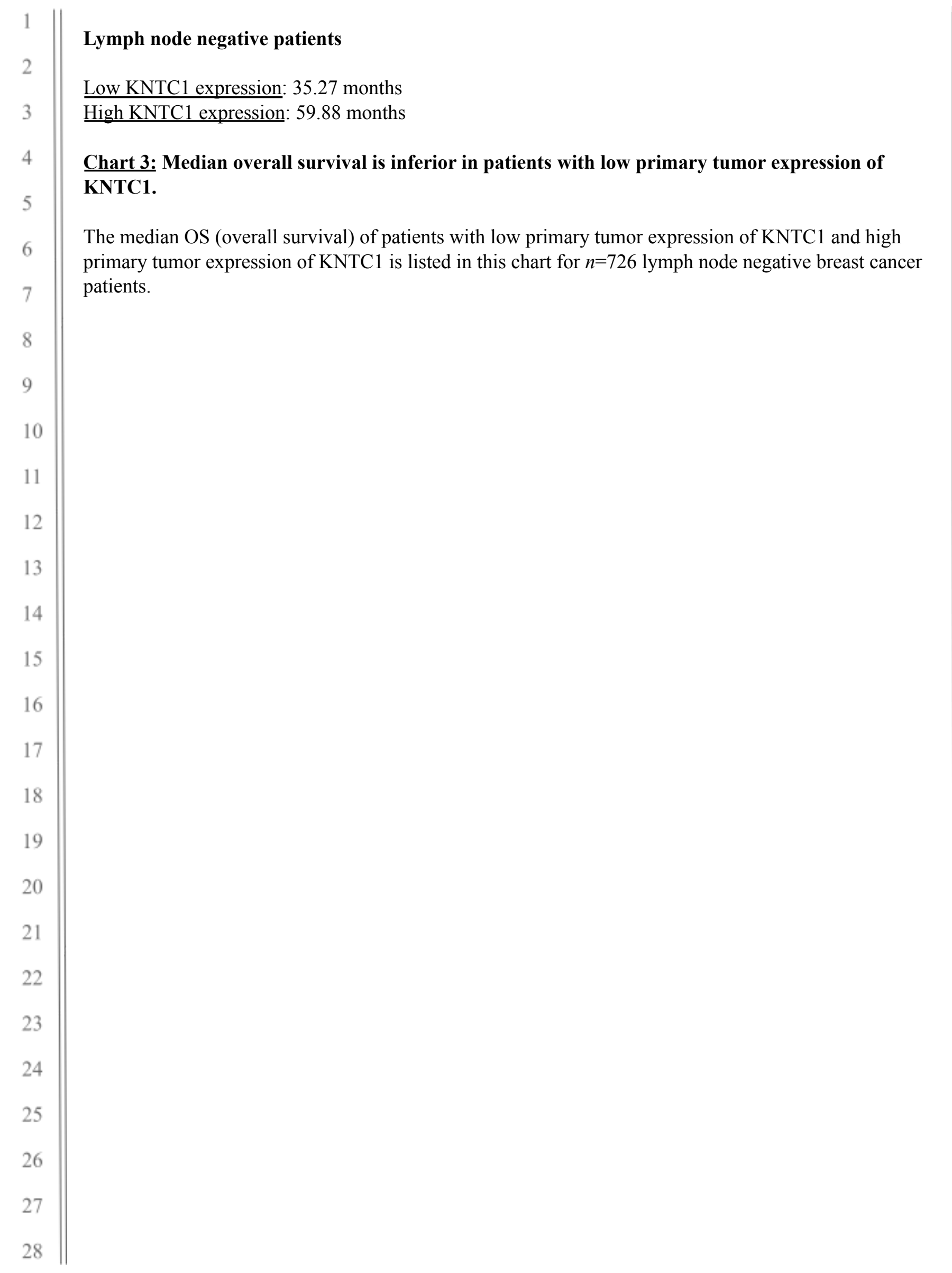

